\title{
Alteration of Interictal Brain Activity in Patients with Temporal Lobe Epilepsy in the Left Dominant Hemisphere: A Resting-State MEG Study
}

\author{
Haitao Zhu, ${ }^{1}$ Jinlong Zhu, ${ }^{1}$ Tiezhu Zhao, ${ }^{2}$ Yong Wu, ${ }^{1}$ Hongyi Liu, ${ }^{1}$ \\ Ting Wu, ${ }^{3}$ Lu Yang, ${ }^{3}$ Yuanjie Zou, ${ }^{1}$ Rui Zhang, ${ }^{1}$ and Gang Zheng ${ }^{2,4}$ \\ ${ }^{1}$ Department of Neurosurgery, Nanjing Brain Hospital Affiliated to Nanjing Medical University, Nanjing, Jiangsu 210029, China \\ ${ }^{2}$ College of Civil Aviation, Nanjing University of Aeronautics and Astronautics, Nanjing, Jiangsu 210016, China \\ ${ }^{3}$ MEG Center, Nanjing Brain Hospital Affiliated to Nanjing Medical University, Nanjing, Jiangsu 210029, China \\ ${ }^{4}$ Department of Medical Imaging, Jinling Hospital, Medical School of Nanjing University, Nanjing, Jiangsu 210002, China
}

Correspondence should be addressed to Rui Zhang; neurosurgeonzr@njmu.edu.cn and Gang Zheng; zg431193@gmail.com

Received 16 May 2014; Revised 3 July 2014; Accepted 5 July 2014; Published 21 July 2014

Academic Editor: Danny Jiongjiong Wang

Copyright ( 2014 Haitao Zhu et al. This is an open access article distributed under the Creative Commons Attribution License, which permits unrestricted use, distribution, and reproduction in any medium, provided the original work is properly cited.

Resting MEG activities were compared between patients with left temporal lobe epilepsy (LTLE) and normal controls. Using SAMg2, the activities of MEG data were reconstructed and normalized. Significantly elevated SAMg2 signals were found in LTLE patients in the left temporal lobe and medial structures. Marked decreases of SAMg2 signals were found in the wide extratemporal lobe regions, such as the bilateral visual cortex. The study also demonstrated a positive correlation between the seizure frequency and brain activities of the abnormal regions after the multiple linear regression analysis. These results suggested that the aberrant brain activities not only were related to the epileptogenic zones, but also existed in other extratemporal regions in patients with LTLE. The activities of the aberrant regions could be further damaged with the increase of the seizure frequency. Our findings indicated that LTLE could be a multifocal disease, including complex epileptic networks and brain dysfunction networks.

\section{Introduction}

Epilepsy is a common neurological disorder, characterized by hypersynchronous neuronal activity as shown from electrophysiological recordings $[1,2]$. Many patients have excellent or good surgical outcomes after the resection of epileptogenic zone. However, the generation and spread of focal onset epileptic seizures involve a large network of brain areas that extended beyond the seizure onset zone (SOZ). Traditionally, the epileptogenic zone was thought to be singular. However, this has been challenged in favor of a network model, in which the focus (or foci) would be widely distributed [3].

During the past decade, there have been an increasing number of studies using structural or functional connectivity methods to research the clinical impact of temporal lobe epilepsy (TLE) on neural networks [4, 5]. Many studies have shown that connectivity abnormalities not only are restricted to the ipsilateral or contralateral temporal lobes, but also involved the extratemporal regions, such as thalamus, cerebellum, frontal lobe areas and cingulate gyrus, and occipital regions $[6,7]$. Activity of the regions functionally or anatomically connected to the temporal lobe or hippocampus probably results in complex cognitive and behavioral conditions. These findings have led to the notion of TLE as a "network disease" [3]. Surgical resection is the gold standard for the localization of SOZ and the evaluation of brain function recovery in TLE patients. However, few cases were confirmed by the surgery and histopathological examinations. Furthermore, the relationship between the epileptogenic zone and other altered brain regions in the TLE network is still unclear.

With the development of medical imaging, there are many advanced image techniques used to study the epileptogenic zone and other brain activities. MEG, a noninvasive detection technology, detects neuronal activity directly with millisecond temporal resolution. Compared 
to electroencephalograph (EEG), which is strongly influenced by conductivity in different organizations within the head, the propagation of magnetic fields is not distorted by the brain, skull, and scalp [8]. Therefore, localizing sources from MEG data is relatively simpler than locating the sources of electric field from EEG data. Previous studies have shown that MEG is a clinically valuable diagnostic tool in presurgical evaluation for both the localization of the epileptogenic zone and the prognosis of surgical outcome $[9,10]$.

In this study, our hypotheses were as follows: (1) the resting-state brain activity may be different across numerous brain regions, rather than only in SOZs, in left temporal lobe epilepsy (LTLE) patients and healthy controls; (2) these differences could be related to the clinical variables of LTLE; (3) the brain abnormalities of LTLE patients could benefit from surgery of the epileptogenic zone. To confirm SOZs, LTLE patients who planned to undergo surgical treatments were included in our study. Factors such as age at onset (year), seizure frequency (per month), and duration of seizure (month) were recorded and followed up with after surgery. To noninvasively assess resting-state brain activity, restingstate MEG (rsMEG) data were acquired in all subjects and quantified based on the SAMg2 method. The SAMg2 values were compared between two groups to detect altered brain regions in LTLE patients and controls. The correlations were calculated to find the relationships between altered brain regions and clinical records of the LTLE patients.

\section{Materials and Methods}

2.1. Subjects. The study was approved by the Medical Ethics Committee of the hospital. Informed consent for the study was obtained from all participants.

From the period of January 2007 to December 2012, 122 patients with refractory epilepsy were admitted to the epilepsy center of the Brain Hospital of Nanjing Medical University (Nanjing, China) and underwent presurgical evaluation. Ninety-eight patients $(80.3 \%)$ ultimately had cortical resection to treat their epilepsy. Twenty LTLE patients (all right-handed, 7 female and 13 male, mean age $25.25 \pm$ $6.90 \mathrm{yrs}$ ) were recruited from the patients who underwent surgical resection for medically intractable epilepsy. Inclusion criteria included (1) seizures with typical temporal lobe semiology that were not controlled with antiepileptic drugs (AEDs); (2) an epileptogenic zone that was located in the left temporal lobe; (3) left hemispheric dominance for language determined by neuropsychological evaluations (etomidate speech and memory test, eSAM, when indicated); (4) patients who underwent surgery for resection of epileptogenic zone; and (5) follow-up time $>12$ months. General information of the patients is summarized in Table 1.

We assessed the clinical factors including the age at onset (year), seizure frequency (per month), and duration of the seizure (month). The seizure frequency was calculated based on the long-term EEG recordings. Two or more seizures were captured during VEEG monitoring. Seizure semiology, ictal, and interictal EEG data were interpreted by an epileptologist and an electrophysiologist to exclude the pseudoepileptic seizures and other interferences. In our study, the mean monitoring time was $257.70 \pm 150.33$ hours, ranging from 96 to 764 hours.

Twenty healthy volunteers (all right-handed, 8 females and 12 males, mean age $25.60 \pm 5.64$ yrs) were recruited as controls, from local community by advertising in the Brain Hospital of Nanjing Medical University. Healthy controls were interviewed and confirmed to have no history of neurological disorders or psychiatric illnesses and no gross abnormalities in brain MRI images. To control the effects of the sleep deprivation in patients, all of our healthy subjects were asked to sleep after 12:00 pm.

\subsection{Methods}

2.2.1. Data Acquisition. All patients had MRI scans with a GE Sigma scanner (GE Healthcare, Milwaukee, WI, USA) before the MEG recording. Three fiducial points were placed in identical locations as the ones used in the MEG recordings so that the 3D MRI and MEG data could be coregistered precisely to yield an MSI using these three landmarks. Three markers, nasion, left ear, and right ear, were stamped on each subject's head. The protocol included the following sequences: axial and sagittal T1 weighted, axial and coronal T2 weighted, axial and coronal fluid-attenuated inversion recovery (FLAIR) images, and three-dimensional (3D) spoiled gradient recalled (SPGR).

rsMEG data acquisitions were performed using a 275channel whole-head system (CTF VSM MedTech Systems Inc., Coquitlam, BC, Canada) in a magnetically shielded room (MSR) (Vacuumschmelze, Hanau, Germany) that was designed to reduce environmental magnetic noise. Before the MEG scan, there was no reduction in the antiepileptic medication due to the potential risk factor. To increase the likelihood of capturing spike events, we used sleep deprivation. The head position relative to the sensor arrays for each patient was measured using three coils affixed to the nasion and preauricular points before MEG data recording. During MEG recording, all subjects were instructed to rest with their eyes closed and heads still. For each subject, 120second MEG data was recorded. If the head movement during the recording was greater than 5 millimeters $(\mathrm{mm})$, the epoch was recorded again.

2.2.2. Data Processing. rsMEG data were preprocessed by CTF software (VSM MedTech Systems Inc., Canada, Version CTF-5.2.1). The frequency ranging from $20 \mathrm{~Hz}$ to $70 \mathrm{~Hz}$ was used in several studies and is outside the range of alphaband activity that would tend to drive the excess kurtosis negative [11-13]. To eliminate the background activity and contrast the interictal spike activity, we performed SAM(g2) analysis localized the epileptic zone in clinical [12, 14, 15]. First, MEG data were filtered $(20-70 \mathrm{~Hz})$. Then, the SAMg2 Z-map of each subject was calculated by the SAMg2 script of the CTF software. After the calculation, the SAMg2 Zmap of each subject was registered with its corresponding 3D MRI by the markers on the nasion, left ear, and right ear. The registered SAMg2 Z-maps were spatially normalized 


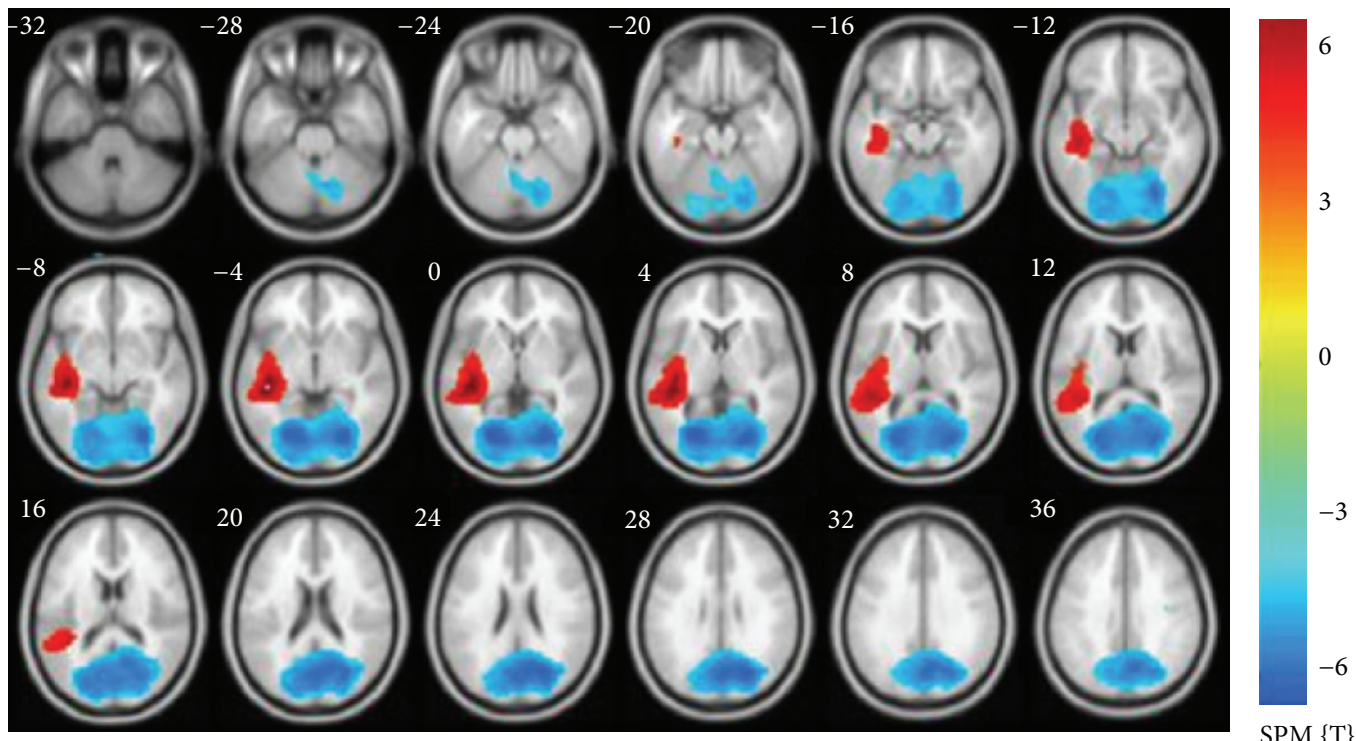

FIGURE 1: Statistical parametric maps depict SAMg2 increases (warm colors) and decreases (cool colors) in LTLE compared with controls. Significant increases occur in left side hippocampal gyrus, insula, superior temporal gyrus, middle temporal gyrus, inferior temporal gyrus, and Heschl gyrus, while decreases occur in the bilateral calcarine, cerebellum, cuneus, fusiform, lingual gyrus, occipital lobe, precuneus, left parietal gyrus, and contralateral precentral gyrus.

to the Montreal Neurological Institute (MNI) template and resampled to $3 * 3 * 3 \mathrm{~mm}^{3}$. Finally, spatial smoothing was conducted on the Z-maps with an isotropic Gaussian kernel of $6 \mathrm{~mm}$ of full width at half maximum.

2.2.3. Statistical Analysis. One way analysis of covariance (ANCOVA) with age and gender as covariates was used to compare SAMg2 maps of healthy controls and those of LTLE patients. The voxel-wise ANCOVA $P$ values were less than 0.05 after false discovery rate (FDR) correction was considered significant.

According to the voxel-wise significant differences between two groups, regions with suprathreshold clusters were defined as regions of interests (ROIs) for further analyses. Mean SAMg2 $Z$ values of these regions were calculated using Automated Anatomical Labeling atlas [16]. Correlations between ROIs and clinical variables of LTLE (seizure onset, seizure frequency, and duration of seizure) were calculated. All data were shown as mean $\pm \mathrm{SD}$. An FDR-corrected $P$ values less than 0.05 were considered significant.

\section{Results}

There was no significant difference in age and gender between the two groups $(P>0.05)$.

3.1. Brain Regions with Abnormal Brain Activity between Two Groups. In the LTLE patients compared to gender and age matched healthy subjects, there was a significant reduction in
SAM g2 signals in the bilateral calcarine, cerebellum, cuneus, fusiform, lingual gyrus, occipital lobe (superior, middle, and inferior gyrus), precuneus, left parietal gyrus, and contralateral precentral gyrus. Patients also showed increased SAM g2 signals in the ipsilateral (left side) hippocampal gyrus, insula, superior temporal gyrus, middle temporal gyrus, inferior temporal gyrus, and Heschl gyri $(P<0.05$, FDR corrected $)$ (Table 2 and Figure 1).

3.2. Correlation Analysis of Abnormal Activity and Clinical Variables. The relationship between the above abnormal regions and clinical variables (age at seizure onset, frequency of seizures, and duration of epilepsy) was evaluated using multiple linear regression analysis. We found that multiple abnormal regions were significantly associated with frequency of seizures (Table 3). Duration of epilepsy and age at seizure onset did not significantly influence brain activity.

\section{Discussion}

In this study, the difference in the SAMg2 signals between patients with LTLE and healthy subjects was used to measure the disruption of neuronal activity in epileptic and distant brain regions during the interictal period. Significant differences were observed in the spatial pattern and intensity of SAMg2 signals in the two groups. These findings are discussed further below. The special changes of the restingstate brain activity may be potential noninvasive biomarkers for understanding, diagnosing, and potentially treating the LTLE patients. 


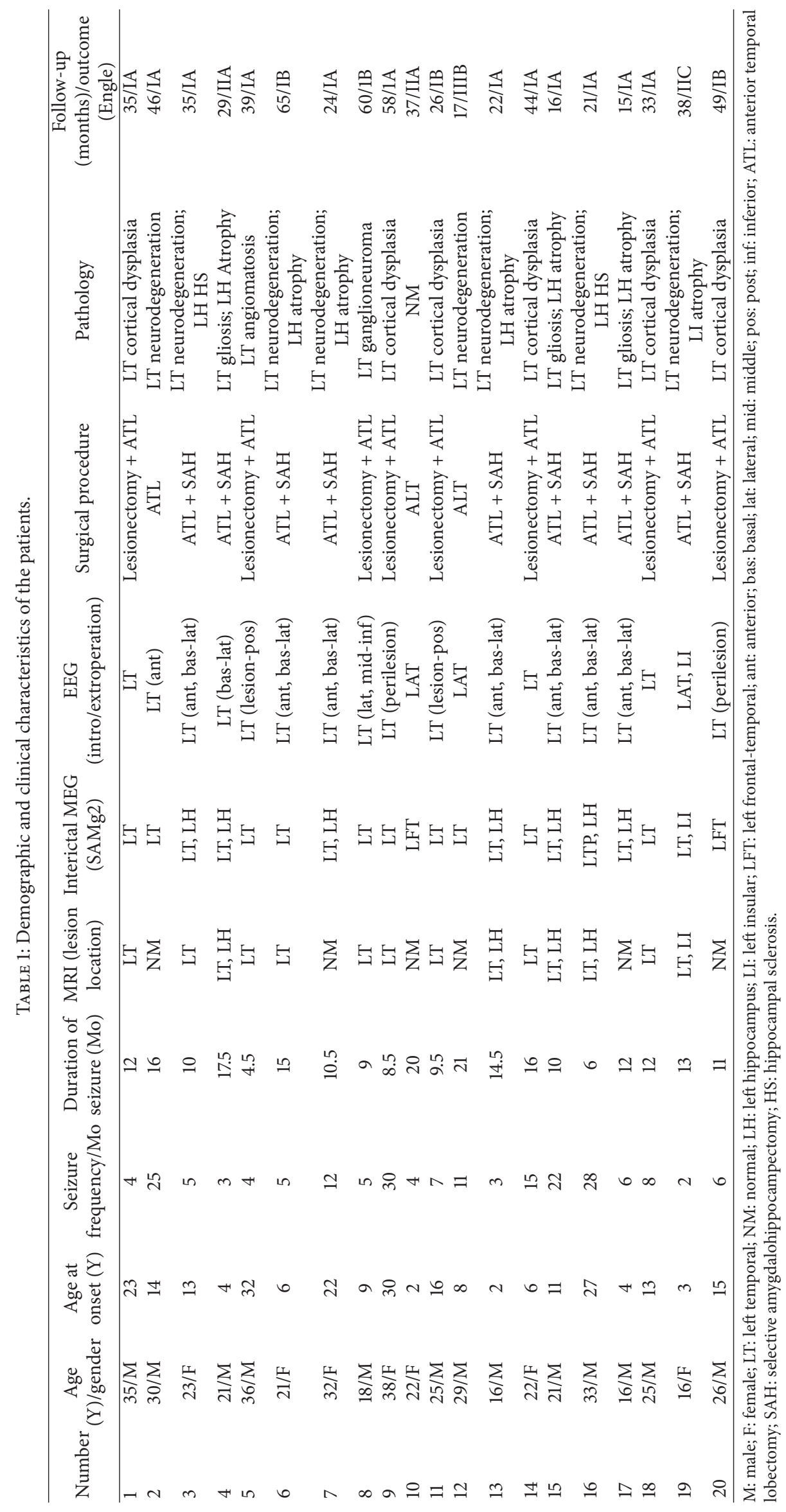


TABLE 2: Brain regions with increased and decreased activations in the patient group.

\begin{tabular}{|c|c|c|c|c|c|}
\hline \multirow{2}{*}{ Brain ROI } & \multirow{2}{*}{ Voxels } & \multirow{2}{*}{$T$ value } & \multicolumn{3}{|c|}{ MNI coordinate } \\
\hline & & & $X$ & $Y$ & $Z$ \\
\hline Temporal_Mid_L & 270 & 4.15 & -48 & -33 & 0 \\
\hline Hippocampus_L & 75 & 4.11 & -36 & -27 & -6 \\
\hline Temporal_Sup_L & 179 & 4.1 & -45 & -36 & 6 \\
\hline Temporal_Inf_L & 36 & 4.04 & -42 & -30 & -12 \\
\hline Heschl_L & 38 & 3.96 & -39 & -21 & 6 \\
\hline Insula_L & 87 & 3.89 & -39 & -18 & 0 \\
\hline Fusiform_L & 92 & -2.03 & -24 & -75 & -3 \\
\hline Precentral_R & 68 & -3.67 & 45 & -3 & 51 \\
\hline Cerebelum_L & 310 & -3.71 & -18 & -72 & -12 \\
\hline Cerebelum_R & 424 & -3.82 & 24 & -81 & -18 \\
\hline Parietal_Sup_L & 38 & -3.86 & -15 & -69 & 42 \\
\hline Precuneus_L & 242 & -4 & 0 & -66 & 30 \\
\hline Occipital_Sup_L & 210 & -4.03 & -18 & -81 & 12 \\
\hline Occipital_Inf_R & 129 & -4.04 & 30 & -81 & -6 \\
\hline Occipital_Inf_L & 115 & -4.06 & -27 & -81 & 0 \\
\hline Occipital_Mid_L & 295 & -4.07 & -27 & -75 & 0 \\
\hline Precuneus_R & 405 & -4.14 & 15 & -66 & 24 \\
\hline Occipital_Mid_R & 262 & -4.15 & 27 & -72 & 27 \\
\hline Lingual_R & 554 & -4.19 & 24 & -75 & -3 \\
\hline Calcarine_L & 533 & -4.3 & -21 & -72 & 6 \\
\hline Occipital_Sup_R & 226 & -4.32 & 27 & -69 & 27 \\
\hline Cuneus_L & 322 & -4.33 & 0 & -72 & 24 \\
\hline Lingual_L & 456 & -4.37 & -21 & -72 & 3 \\
\hline Fusiform_R & 159 & -4.44 & 27 & -75 & -6 \\
\hline Calcarine_R & 474 & -4.44 & 24 & -75 & 9 \\
\hline Cuneus_R & 335 & -4.49 & 21 & -69 & 27 \\
\hline
\end{tabular}

L: left; R: right; Mid: middle; Sup: superior; Inf: inferior.

Compared to the healthy control group, SAMg2 values increased in the ipsilateral (left side) hippocampal gyrus, insula, superior temporal gyrus, middle temporal gyrus, inferior temporal gyrus, and Heschl gyri of the patients. These results revealed that the irritated areas were beyond the $\mathrm{SOZ}$ but restricted to the ipsilateral temporal lobe and mesial structure. We propose that the increased $\mathrm{SAMg} 2$ signals in specific regions may form irritated areas that might be responsible for seizure genesis and propagation. The removal of the irritated areas may be an effective treatment for the LTLE patients.

The role of the neurosurgeon in the surgical treatment of TLE had evolved from doing so-called standard anterior temporal lobectomies (TLR) to selective amygdalohippocampectomy (SAH) and temporal resection guided by intraoperative electrocorticography. A number of studies were devoted to the comparison of seizure results from temporal lobectomy (TLR) with selective amygdalohippocampectomy (SAH) [17, 18]. And several studies have reported that seizure outcomes are irrespective of the extent of mesial and lateral resection $[19,20]$, while others state that seizure outcomes are better if larger volumes or specific substructures are removed [21].
TABLE 3: Results of correlation analysis between the abnormal activity and the seizure frequency ( $P<0.05$ with FDR correction).

\begin{tabular}{lcc}
\hline Brain ROI & $P$ value & $R$ value \\
\hline Precentral_R & 0.025 & 0.377 \\
Hippocampus_L & 0.025 & 0.406 \\
Calcarine_L & 0.025 & 0.373 \\
Calcarine_R & 0.027 & 0.349 \\
Cuneus_L & 0.021 & 0.474 \\
Cuneus_R & 0.026 & 0.357 \\
Lingual_R & 0.026 & 0.409 \\
Occipital_Sup_L & 0.031 & 0.488 \\
Occipital_Sup_R & 0.021 & 0.454 \\
Occipital_Mid_L & 0.025 & 0.383 \\
Occipital_Mid_R & 0.040 & 0.520 \\
Occipital_Inf_L & 0.022 & 0.441 \\
Occipital_Inf_R & 0.023 & 0.478 \\
Fusiform_L & 0.027 & 0.481 \\
Fusiform_R & 0.026 & 0.388 \\
Parietal_Sup_L & 0.022 & 0.431 \\
Precuneus_L & 0.022 & 0.459 \\
Precuneus_R & 0.027 & 0.389 \\
Temporal_Mid_L & 0.024 & 0.370 \\
Temporal_Inf_L & 0.033 & 0.577 \\
Cerebelum_L & 0.035 & 0.313 \\
Cerebelum_R & 0.028 & 0.517 \\
\hline : & &
\end{tabular}

L: left; R: right; Mid: middle; Sup: superior; Inf: inferior.

Comparing TLR and SAH, McKhann pointed out, "neither SAH nor TLR could be recommended over the other option as a standard or guideline in the surgical management of TLE" [22]. The Spencer type of resection [23], which consists of a small anterior partial lobectomy combined with a more extensive mesial resection, may be an ideal method for the treatment of TLE. Our study reveals that the irritated areas of LTLE involve not only the lateral neocortical structures but also the ipsilateral nearby structures, such as the hippocampus (HC), insula, and Heschl gyri. These interictal irritated zones may be involved in the LTLE network formation. The Spencer type of resection would be sufficient enough to resect or interrupt a large enough part of the mesial structures to render the mesiobasal network between the $\mathrm{HC}$, parahippocampal gyrus, and amygdalum unable to build up and sustain a seizure. Abosch et al. [24] referred to this as "severing a critical proportion of the connections" when they discussed the variability in extent of resection described by McKhann et al. [22].

In this study, all of the patients were diagnosed with LTLE and eleven of them underwent the Spencer type of resection (left anterior temporal lobectomy combined with partial amygdalohippocampectomy). The seizure freedom (Engel class I) of them was 72.7\% (8/11) better than the group of the remainder $55.6 \%(5 / 9)$. The results from this study have further proved that the refractory LTLE is not a focal disease but a network disease and can be cured by extensive surgical resection. 
The appearance of the insula is also significant because of its particular interconnection with the amygdalohippocampal structures. Isnard et al. found that seizure activity invaded the insular cortex in all temporal lobe epilepsy patients studied with chronic depth stereotactic recordings [25]. This may explain the persistence of seizures after selective amygdalohippocampectomy. This study also had its own limitations; we have removed the anterior temporal lobe and the mesial structures except the insula during surgery.

The reduction in SAMg2 values is thought to reflect deactivated brain activity or dysfunction in the special regions. Compared with healthy controls, LTLE patients showed multifocal dysfunction in the bilateral calcarine, cerebellum, cuneus, fusiform, lingual gyrus, occipital lobe (superior, middle, and inferior gyrus), and precuneus brain regions. The ipsilateral superior parietal gyrus and contralateral precentral gyrus also displayed reduction. The deactivated areas extend widely beyond the ipsilateral hemisphere to the contralateral or bilateral areas in patients with LTLE.

A relatively large dysfunctional area was observed in the bilateral visual cortex, including the bilateral calcarine, fusiform, lingual gyrus, and occipital lobe (superior, middle, and inferior gyrus) in this study. Van Paesschen et al. used SPECT to study the cerebral perfusion changes in complex partial seizures patients with hippocampal sclerosis. They found bilateral occipital hyperperfusion during complex partial seizure and relative occipital hypoactivation during interictal period [26].

The cause of interictal bilateral visual cortex dysfunction is unknown, but propagating epileptiform discharges through the branches of the inferior longitudinal fasciculus (ILF) may play a role. Catani et al. [27] used tractography in the living human brain to address the connections between occipital and temporal lobe and found direct fiber connections between the occipital and anterior temporal cortex in a bundle labeled the ILF. In our study, the dysfunctional regions also involved the three origin branches of the ILF: a lingual branch, a lateral occipital branch, and a cuneal branch. Moreover, a recent study combining fMRI and tractography has visualized the propagation of epileptic activity from the temporal epileptogenic focus to the occipital lobe in mesial temporal lobe epilepsy [28]. The involved cortices lie along the occipitotemporal connections supplied by the inferior longitudinal fasciculus, suggesting a direct propagation pathway from the anterotemporal to the occipital lobe [29]. However, in line with previous brain imaging studies we take our data to suggest that the left side epileptic interictal discharges may influence the activity of the bilateral visual cortex in the patients with left temporal lobe epilepsy.

Our study just focuses on the reduced active regions of the default mode network (DMN) within the bilateral precuneus, ipsilateral superior parietal gyrus, while the other regions of the DMN are normal. Decreased SAMg2 signals within the DMN are considered to result from the disruption of neuronal activity and are commonly used to reflect associated impairments in brain disorders. The lateral parietal cortex is involved in spatial attention aspects of word reading [30]. The precuneus (PCUN) has been reported to be involved in consciousness, engaged in self-related mental representations during rest [31], and related to the late recovery of consciousness in epilepsy patients [32]. The posterior cingulate cortex/precuneus (PCC/ PCUN) is activated during tasks that involve autobiographic memory and self-referential processes [33]. TLE patients often present a few abnormal psychological and psychiatric symptoms associated with the functionalities of the DMN [34], such as absence of selfawareness, emotional and psychic experiences, and social cognitive impairments.

The motor impairment of LTLE patients has been demonstrated by this study. Our MEG study revealed that the deactivated region involved the contralateral precentral gyrus and the bilateral cerebellum. The contralateral precentral gyrus is dominated by the control of limb movement ipsilateral to the epileptic focus, while the cerebellum is by sensory-motor integration, motor coordination, and so forth. Nelissen et al., using SPECT and PET to study the brain blood perfusion in TLE, found interictal hypometabolism in the frontal lobe cortex. They suggested a dynamic process of frontal lobe function inhibition, which not only could represent protection against epileptiform-discharge propagation, but could also be responsible for the functional deficits presented by these patients. In this study, we also found more serious damages in bilateral cerebellum lobe in our LTLE patients. This phenomenon was so-called "crossed cerebellar diaschisis" and was considered an indication of disconnection of the glutamatergic corticopontocerebellar tracts [35]. The decreased SAMg2 values of the bilateral cerebellum lobe could be a sign of decreased motor coordination.

In our study, sleep deprivation was used to increase the likelihood of capturing spike events which were performed in all epilepsy patients in our epilepsy center. Many studies also adopted the similar sleep deprivation method to increase the likelihood of capturing spike events in clinical $[36,37]$. A lack of sleep has been demonstrated to produce performance deficits in experimental tasks of alertness, attention, memory, cognition, learning, and motor responses [38]. While the neurophysiological effects of sleep deprivation remain incompletely understood, a few recent studies have begun to provide guidance on where and what neurophysiological changes occur as a function of sleep deprivation. Thomas et al. quantified and characterized global and regional brain activity changes implicated in sleep deprivation-induced neurobehavioral impairment during cumulative, extended sleep loss [39]. Significant decreases in CMRglu were reported in the thalamus, prefrontal and posterior parietal cortices. Alertness and cognitive performance declined in association with these brain deactivations. This study provides evidence that short-term sleep deprivation produces global and regional decreases in brain activity, with larger reductions in activity in the distributed cortical-thalamic network mediating attention and higher-order cognitive processes. The results from sleep deprivation were different than the hyperactivity and dysfunctional regions which were due to epilepsy in our study. In our study, the healthy volunteers were demanded to sleep after twelve o'clock before MEG examinations to control the additional effects of the sleep loss in LTLE patients. The decreased brain activities detected in our patients were caused by the long-term effects of epilepsy 
rather than sleep loss. Considering the complex effects of sleep deprivation on brain activity, further study should be performed to control sleep loss in patients with epilepsy.

In our study, we used the SAMg2 signals to study how brain activity in LTLE patients differs from brain activity in healthy controls. Specifically, seizure frequency was strongly associated with intensity of abnormal activity in the LTLE patients. In the study, we further confirmed that TLE is not a focal focus, but a multifocal disease. The mechanism of these extensive abnormal activities needs further study.

\section{Limitations}

In the study, we did not compare the preoperative brain activities of TLE with the changes of postoperative patients. Further, this study was limited by not comparing abnormal activities with neuropsychological findings and prognostic factor. To make up the difference between TLE patients and controls in the sleep loss, all our health subjects were asked to sleep later. Though sleep loss was not totally controlled, our findings were quite different from those in sleep deprivation, indicating that our study was less affected by sleep deprivation. However, it is difficult to exclude the potential impact of the sleep deprivation to the brain activity of the patients and the normal subjects. Further study should be conducted to specify the effects of sleep loss in TLE patients.

\section{Conflict of Interests}

The authors disclose no conflicts.

\section{Acknowledgments}

This paper is supported by Grants from National Natural Science Foundation of China (Grant no. 81101039 to G. Zheng) and from Natural Science Foundation of Jiangsu Province (Grant no. BK20131081 to R. Zhang).

\section{References}

[1] C. A. Schevon, J. Cappell, R. Emerson et al., "Cortical abnormalities in epilepsy revealed by local EEG synchrony," NeuroImage, vol. 35, no. 1, pp. 140-148, 2007.

[2] G. J. Ortega, L. Menendez de la Prida, R. G. Sola, and J. Pastor, "synchronization clusters of interictal activity in the lateral temporal cortex of epileptic patients: Intraoperative electrocorticographic analysis," Epilepsia, vol. 49, no. 2, pp. 269280, 2008.

[3] L. Bonilha, T. Nesland, G. U. Martz et al., "Medial temporal lobe epilepsy is associated with neuronal fibre loss and paradoxical increase in structural connectivity of limbic structures," Journal of Neurology, Neurosurgery and Psychiatry, vol. 83, no. 9, pp. 903-909, 2012.

[4] G. Bettus, E. Guedj, F. Joyeux et al., "Decreased basal fMRI functional connectivity in epileptogenic networks and contralateral compensatory mechanisms," Human Brain Mapping, vol. 30, no. 5, pp. 1580-1591, 2009.

[5] G. Doucet, K. Osipowicz, A. Sharan, M. R. Sperling, and J. I. Tracy, "Extratemporal functional connectivity impairments at rest are related to memory performance in mesial temporal epilepsy," Human Brain Mapping, vol. 34, no. 9, pp. 2202-2216, 2013.

[6] N. Bernasconi, S. Duchesne, A. Janke, J. Lerch, D. L. Collins, and A. Bernasconi, "Whole-brain voxel-based statistical analysis of gray matter and white matter in temporal lobe epilepsy," NeuroImage, vol. 23, no. 2, pp. 717-723, 2004.

[7] F. Riederer, R. Lanzenberger, M. Kaya, D. Prayer, W. Serles, and C. Baumgartner, "Network atrophy in temporal lobe epilepsy: a voxel-based morphometry study," Neurology, vol. 71, no. 6, pp. 419-425, 2008.

[8] M. S. Hamalainen, "Magnetoencephalography: a tool for functional brain imaging," Brain Topography, vol. 5, no. 2, pp. 95-102, 1992.

[9] R. Ramachandrannair, H. Otsubo, M. M. Shroff et al., "MEG predicts outcome following surgery for intractable epilepsy in children with normal or nonfocal MRI findings," Epilepsia, vol. 48, no. 1, pp. 149-157, 2007.

[10] M. J. M. Fischer, G. Scheler, and H. Stefan, "Utilization of magnetoencephalography results to obtain favourable outcomes in epilepsy surgery," Brain, vol. 128, no. 1, pp. 153-157, 2005.

[11] R. Ishii, L. Canuet, A. Ochi et al., "Spatially filtered magnetoencephalography compared with electrocorticography to identify intrinsically epileptogenic focal cortical dysplasia," Epilepsy Research, vol. 81, no. 2-3, pp. 228-232, 2008.

[12] H. E. Kirsch, S. E. Robinson, M. Mantle, and S. Nagarajan, "Automated localization of magnetoencephalographic interictal spikes by adaptive spatial filtering," Clinical Neurophysiology, vol. 117, no. 10, pp. 2264-2271, 2006.

[13] D. F. Rose, H. Fujiwara, K. Holland-Bouley, H. M. Greiner, T. Arthur, and F. T. Mangano, "Focal peak activities in spread of interictal-ictal discharges in epilepsy with beamformer meg: Evidence for an epileptic network?" Frontiers in Neurology, vol. 4, article 56, Article ID Article 56, 2013.

[14] S. E. Robinson, S. S. Nagarajan, M. Mantle, V. Gibbons, and H. Kirsch, "Localization of interictal spikes using $\operatorname{SAM}(g 2)$ and dipole fit," Neurology \& Clinical Neurophysiology, vol. 2004, article 74, 2004.

[15] I. Sugiyama, K. Imai, Y. Yamaguchi et al., "Localization of epileptic foci in children with intractable epilepsy secondary to multiple cortical tubers by using synthetic aperture magnetometry kurtosis," Journal of Neurosurgery: Pediatrics, vol. 4, no. 6, pp. 515-522, 2009.

[16] N. Tzourio-Mazoyer, B. Landeau, D. Papathanassiou et al., "Automated anatomical labeling of activations in SPM using a macroscopic anatomical parcellation of the MNI MRI singlesubject brain," NeuroImage, vol. 15, no. 1, pp. 273-289, 2002.

[17] E. Paglioli, A. Palmini, M. Portuguez et al., "Seizure and memory outcome following temporal lobe surgery: selective compared with nonselective approaches for hippocampal sclerosis," Journal of Neurosurgery, vol. 104, no. 1, pp. 70-78, 2006.

[18] H. Bate, P. Eldridge, T. Varma, and U. C. Wieshmann, "The seizure outcome after amygdalohippocampectomy and temporal lobectomy," European Journal of Neurology, vol. 14, no. 1, pp. 90-94, 2007.

[19] V. Salanova, O. Markand, and R. Worth, "Temporal lobe epilepsy: analysis of failures and the role of reoperation," Acta Neurologica Scandinavica, vol. 111, no. 2, pp. 126-133, 2005.

[20] M. J. Hennessy, R. D. C. Elwes, C. D. Binnie, and C. E. Polkey, "Failed surgery for epilepsy: a study of persistence and recurrence of seizures following temporal resection," Brain, vol. 123, no. 12, pp. 2445-2466, 2000. 
[21] C. Tonini, E. Beghi, A. T. Berg et al., "Predictors of epilepsy surgery outcome: a meta-analysis," Epilepsy Research, vol. 62, no. 1, pp. 75-87, 2004.

[22] G. M. McKhann II, J. Schoenfeld-McNeill, D. E. Born, M. M. Haglund, and G. A. Ojemann, "Intraoperative hippocampal electrocorticography to predict the extent of hippocampal resection in temporal lobe epilepsy surgery," Journal of Neurosurgery, vol. 93, no. 1, pp. 44-52, 2000.

[23] D. D. Spencer, S. S. Spencer, R. H. Mattson, P. D. Williamson, and R. A. Novelly, "Access to the posterior medial temporal lobe structures in the surgical treatment of temporal lobe epilepsy," Neurosurgery, vol. 15, no. 5, pp. 667-671, 1984.

[24] A. Abosch, N. Bernasconi, W. Boling et al., "Factors predictive of suboptimal seizure control following selective amygdalohippocampectomy," Journal of Neurosurgery, vol. 97, no. 5, pp. 11421151, 2002.

[25] J. Isnard, M. Guenot, K. Ostrowsky, M. Sindou, and F. Mauguiere, "The role of the insular cortex in temporal lobe epilepsy," Annals of Neurology, vol. 48, pp. 614-623, 2000.

[26] W. Van Paesschen, P. Dupont, G. van Driel, H. van Billoen, and A. Maes, "SPECT perfusion changes during complex partial seizures in patients with hippocampal sclerosis," Brain, vol. 126, no. 5, pp. 1103-1111, 2003.

[27] M. Catani, D. K. Jones, R. Donato, and D. H. Ffytche, "Occipitotemporal connections in the human brain," Brain, vol. 126, no. 9, pp. 2093-2107, 2003.

[28] K. Hamandi, H. W. R. Powell, H. Laufs et al., "Combined EEGfMRI and tractography to visualise propagation of epileptic activity," Journal of Neurology, Neurosurgery and Psychiatry, vol. 79, no. 5, pp. 594-597, 2008.

[29] R. Wiest, L. Estermann, O. Scheidegger et al., "Widespread grey matter changes and hemodynamic correlates to interictal epileptiform discharges in pharmacoresistant mesial temporal epilepsy," Journal of Neurology, vol. 260, no. 6, pp. 1601-1610, 2013.

[30] T. Bitan, D. Manor, I. A. Morocz, and A. Karni, "Effects of alphabeticality, practice and type of instruction on reading an artificial script: an fMRI study," Cognitive Brain Research, vol. 25, no. 1, pp. 90-106, 2005.

[31] A. E. Cavanna and M. R. Trimble, "The precuneus: a review of its functional anatomy and behavioural correlates," Brain, vol. 129, no. 3, pp. 564-583, 2006.

[32] P. Dupont, J. J. Zaknun, A. Maes et al., "Dynamic perfusion patterns in temporal lobe epilepsy," European Journal of Nuclear Medicine and Molecular Imaging, vol. 36, no. 5, pp. 823-830, 2009.

[33] R. L. Buckner and D. C. Carroll, "Self-projection and the brain," Trends in Cognitive Sciences, vol. 11, pp. 49-57, 2007.

[34] R. L. Buckner, J. R. Andrews-Hanna, and D. L. Schacter, "The brain's default network: anatomy, function, and relevance to disease," Annals of the New York Academy of Sciences, vol. 1124, pp. 1-38, 2008.

[35] N. Nelissen, W. van Paesschen, K. Baete et al., "Correlations of interictal FDG-PET metabolism and ictal SPECT perfusion changes in human temporal lobe epilepsy with hippocampal sclerosis," NeuroImage, vol. 32, no. 2, pp. 684-695, 2006.

[36] Z. Xiao, J. Xiang, S. Holowka et al., "Volumetric localization of epileptic activities in tuberous sclerosis using synthetic aperture magnetometry," Pediatric Radiology, vol. 36, no. 1, pp. 16-21, 2006.
[37] J. S. Archer, D. F. Abbott, A. B. Waites, and G. D. Jackson, "fMRI "deactivation" of the posterior cingulate during generalized spike and wave," NeuroImage, vol. 20, no. 4, pp. 1915-1922, 2003.

[38] Y. Harrison and J. A. Horne, "The impact of sleep deprivation on decision making: a review," Journal of Experimental Psychology: Applied, vol. 6, no. 3, pp. 236-249, 2000.

[39] M. Thomas, H. Sing, G. Belenky et al., "Neural basis of alertness and cognitive performance impairments during sleepiness. I. Effects of $24 \mathrm{~h}$ of sleep deprivation on waking human regional brain activity," Journal of Sleep Research, vol. 9, no. 4, pp. 335$352,2000$. 


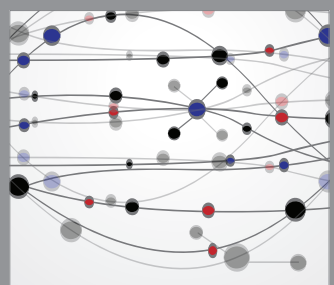

The Scientific World Journal
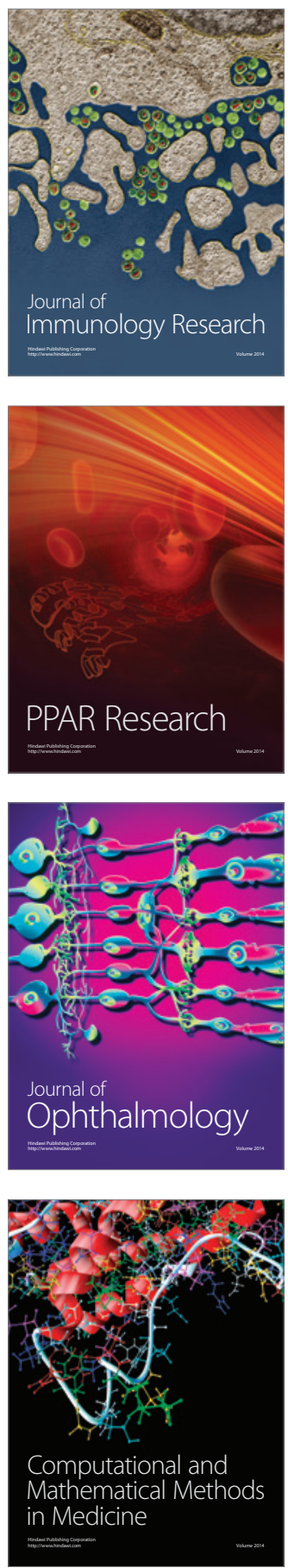

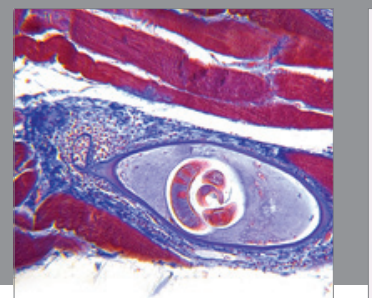

Gastroenterology

Research and Practice
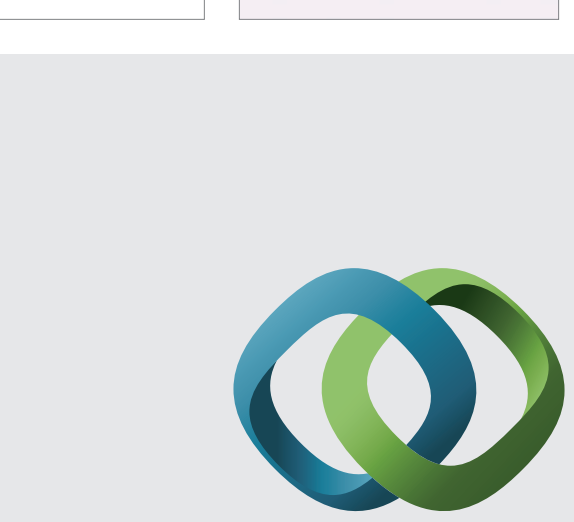

\section{Hindawi}

Submit your manuscripts at

http://www.hindawi.com
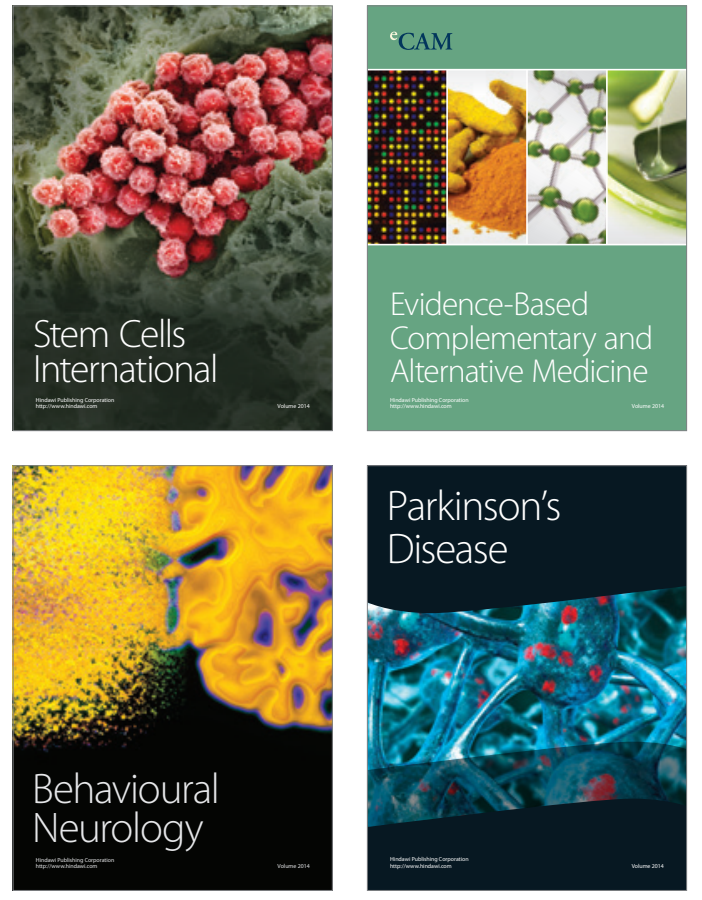
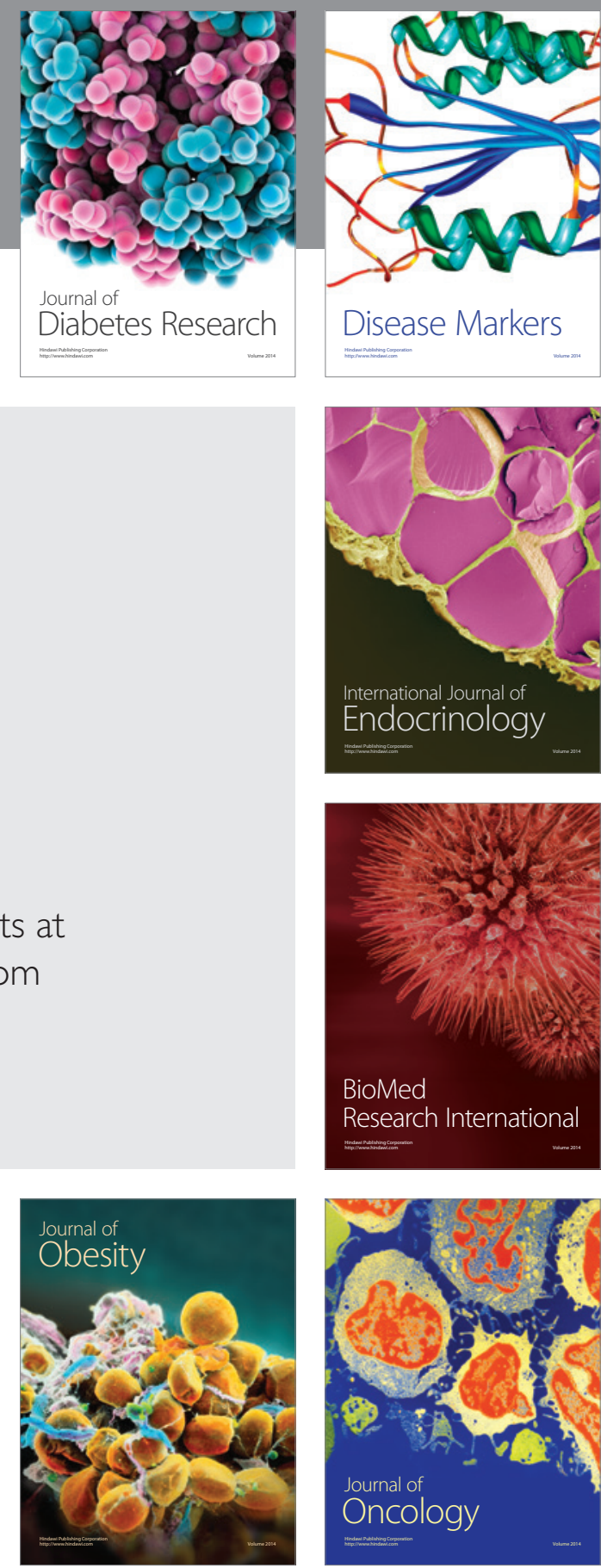

Disease Markers
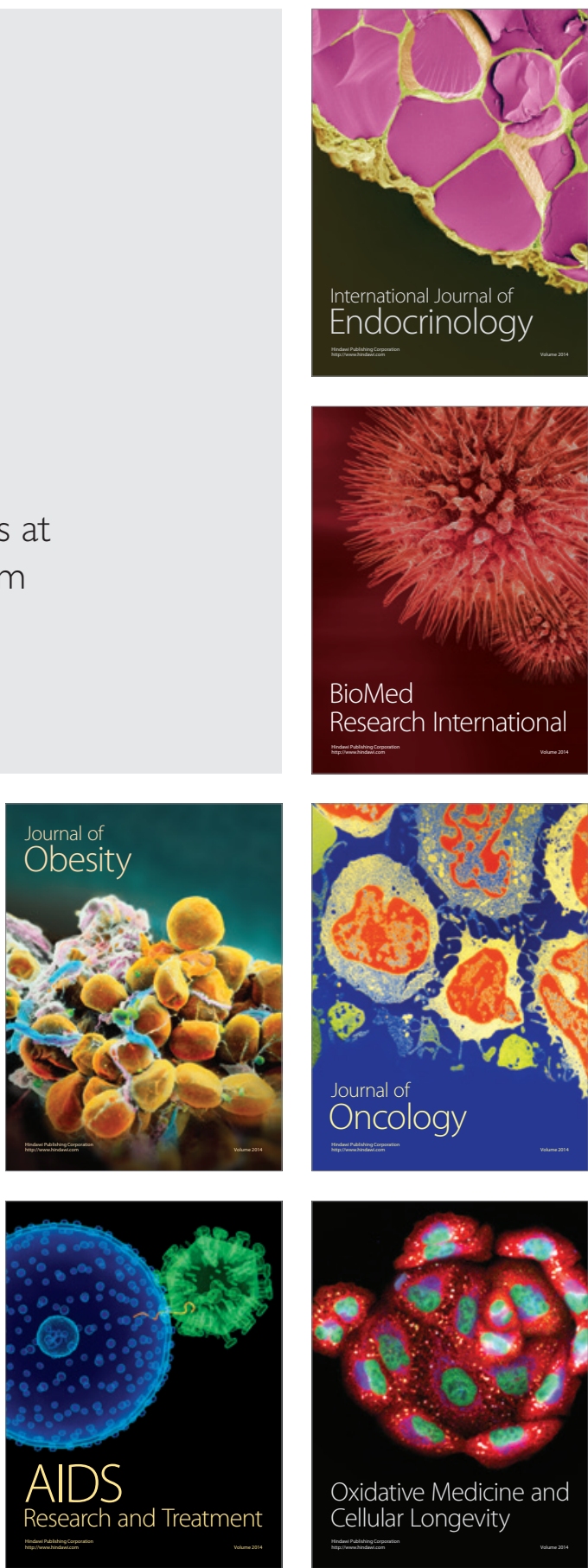